\title{
Risk factors for caesarean section in primigravidae
}

\author{
Preeti Malapure, Sumayya, Rajeshwari P, Sreelatha S \\ Correspondence: Prof (Dr) Sreelatha S, Professor in Department of Obstetrics and \\ Gynaecology, PGIMSR Rajajinagar, Bangalore, Karnataka; Email - \\ dr.sreelatha2011@gmail.com
}

Distributed under Attribution-Non Commercial-Share Alike 4.0 International (CC BY-NC-SA 4.0)

\begin{abstract}
Objectives: To determine the antepartum and intrapartum risk factors leading to caesarean section in Primigravidae. Methods: A prospective study of 100 patients conducted in the department of Obstetrics and Gynaecology from January 2018 to June 2018. Data collected included the age of the patient, obstetric history, gestational age, clinical symptoms, antepartum and intrapartum risk factors for LSCS. Results: The most common antepartum risk factor for caesarean section in primigravidae was found to be fetal distress (41\%) and the most common intrapartum finding was meconium stained liquor (27\%). Conclusion: Presence of risks complicating pregnancies has increased the rates of primary caesarean section. It is prudent to carefully weigh the risks and benefits associated with caesarean section in order to improve the pregnancy outcome.
\end{abstract}

Keywords: Primigravida, caesarean section, fetal distress, meconium stained liquor, cardiotocography.

Caesarean section delivery is a life-saving surgical procedure that is necessary when certain fetal and/or maternal complications arise during pregnancy or labour. Since 1985, healthcare community worldwide has decided that the ideal rate for caesarean sections should be between $10-15 \%{ }^{1}$. However in the past few decades, caesarean section rates have substantially increased due to various causes and it is important to accurately identify the reasons for this increased trend. Caesarean section in a primigravidae also increases the morbidity for the next pregnancy turning her into an obstetric cripple. Hence identifying the antepartum and postpartum risk factors leading to primary caesarean section is important. Fetal distress is a common terminology used as an indication for lower segment caesarian section (LSCS). The availability of intrapartum cardiotocographic monitoring and antenatal doppler flow studies of the umbilical and other fetal vessels have improved diagnosis of the fetuses at risk to develop fetal distress. ${ }^{2}$ Complications can occur unexpectedly even in a low risk patient and thus presence of well trained staff in a well equipped centre is required. This study aims to identify the antepartum and intrapartum risk factors involved in caesarean section in primigravidae.

\section{Methods}

This was a prospective study conducted in the department of Obstetrics and Gynaecology in ESIC-MCPGIMSR, Bangalore for duration of 6 months from January 2018 to June 2018. A total of 100 patients were included who underwent primary caesarean section and

Received: $21^{\text {th }}$ August 2018. Accepted: $1^{\text {st }}$ January 2019.

Malapure P, Sumayya, Rajeshwari P, Sreelatha S. Risk factors for caesarean section in primigravidae. The New Indian Journal of OBGYN. 2019; 6(1):32-5. 
The New Indian Journal of OBGYN. 2019 (July-December); 6(1)

data were collected including the age of the patient, obstetric history, gestational age, clinical symptoms, antepartum and intrapartum risk factors for LSCS, diagnosis, and maternal outcome. Data were expressed in tables as number and percentage.

\section{Results}

Among the 100 primigravida patients, majority of the patients (87\%) were in the age group 20-29 years. Eighty eight $(88 \%)$ cases were full term patients and $12 \%$ cases

Table 1: Age and Period of gestation

\begin{tabular}{lll}
\hline Categories & & Number (\%) \\
\hline Age distribution in & $<20$ & $1(1 \%)$ \\
years & $20-25$ & $47(47 \%)$ \\
& $26-29$ & $40(40 \%)$ \\
& $30-34$ & $11(11 \%)$ \\
Period of gestation & $>35$ & $1(1 \%)$ \\
in weeks & $<37$ & $12(12 \%)$ \\
\hline
\end{tabular}

were preterm (Table 1). The most common indication for LSCS was fetal distress in $41 \%$ cases which included non reassuring non stress test (NST) and thick meconium stained liquor. Severe oligohydramnios was the indication

Table 2: Indications for LSCS

\begin{tabular}{ll}
\hline Indications & Number (\%) \\
\hline Fetal distress & $41(41 \%)$ \\
Prolonged PROM & $6(6 \%)$ \\
PPROM & $2(2 \%)$ \\
Deep transverse arrest & $4(4 \%)$ \\
Malpresentation & $1(1 \%)$ \\
Cephalo pelvic disproportion & $6(6 \%)$ \\
Failed induction of labour & $9(9 \%)$ \\
Abnormal doppler & $2(2 \%)$ \\
Severe oligohydramnios & $10(10 \%)$ \\
Breech & $6(6 \%)$ \\
Second stage arrest of cervical dilation & $1(1 \%)$ \\
Cord presentation & $1(1 \%)$ \\
Non progress of labour & $1(1 \%)$ \\
Severe IUGR & $2(2 \%)$ \\
Persistent right occipito-posterior & $1(1 \%)$ \\
Eclampsia & $1(1 \%)$ \\
Arrest of descent & $1(1 \%)$ \\
Placenta previa & $1(1 \%)$ \\
Hand prolapse & $1(1 \%)$ \\
\hline
\end{tabular}

for LSCS in $10 \%$ cases. The other indications included breech $(6 \%)$, prolonged premature rupture of membrane $(\mathrm{PROM})(6 \%)$, preterm premature rupture of membrane (PPROM) (2\%), deep transverse arrest (4\%), failed induction of labour (9\%), malpresentation (1\%), cephalopelvic disproportion ( $6 \%)$, abnormal doppler
Table 3: Antepartum risk factors

\begin{tabular}{ll}
\hline Risk factors & Number (\%) \\
\hline No comorbidities & $39(39 \%)$ \\
Oligohydramnios & $25(25 \%)$ \\
Hypothyroidism & $14(14 \%)$ \\
Hypertensive disorders of pregnancy & $10(10 \%)$ \\
PROM & $9(9 \%)$ \\
PPROM & $4(4 \%)$ \\
Anemia & $4(4 \%)$ \\
IUGR & $1(1 \%)$ \\
Diabetes mellitus & $1(1 \%)$ \\
Rh negative pregnancy & $2(2 \%)$ \\
IVF conception & $1(1 \%)$ \\
Seizure disorder & $1(1 \%)$ \\
Asthma & $1(1 \%)$ \\
Bad obstetric history & $1(1 \%)$ \\
HBsAg positive & $2(2 \%)$ \\
Ovarian cyst & $1(1 \%)$
\end{tabular}

(2\%), second stage arrest of cervical dilation (1\%), cord presentation(1\%), non progress of labour(1\%), severe intra uterine growth restriction (IUGR) (2\%), persistent right occipito-posterior( $1 \%)$, eclampsia( $1 \%$ ), arrest of descent $(1 \%)$, placenta previa $(1 \%)$ and hand prolapse $(1 \%)$ (Table 2).

The most common antepartum risk factor for LSCS in primigravidae was oligohydramnios $(25 \%)$. However no comorbidities were seen in the majority (39\%) cases.

Table 4: Risk factors

\begin{tabular}{ll}
\hline Risk factors & Number (\%) \\
\hline Meconium stained liquor & $27(27 \%)$ \\
PROM & $2(2 \%)$ \\
Loop of cord around neck & $11(11 \%)$ \\
Right occipito posterior & $1(1 \%)$ \\
Deep transverse arrest & $1(1 \%)$ \\
Decreased liquor & $14(14 \%)$ \\
Deflexed head & $3(3 \%)$ \\
Fibroid uterus & $1(1 \%)$ \\
Right ovarian cyst & $1(1 \%)$ \\
No abnormalities detected & $25(25 \%)$
\end{tabular}

Hypothyroidism was seen in $14 \%$ cases and $10 \%$ cases had hypertensive disorders of pregnancy. Other risk factors included PROM (9\%), PPROM (4\%), anemia (4\%), $\mathrm{Rh}$ negative pregnancy ( $2 \%$ ), Hbs Ag positive in $2 \%$ cases and one each of IUGR, diabetes mellitus, seizure disorder, bronchial asthma, bad obstetric history and 
ovarian cyst. Some patients had more than one associated risk factors (Table 3 ).

The most common intra operative finding was meconium stained liquor (27\%), loop of cord around neck $(11 \%)$ and scanty liquor in $14 \%$ cases. However no abnormalities were seen in $25 \%$ cases (Table 4).

\section{Discussion}

This prospective study was conducted over a period of 6 months to see the antepartum and postpartum risk factors involved in caesarean section in primigravidae patients. In the study by Kohli et al ${ }^{3}$ majority of the patients (77.7\%) undergoing emergency caesarean section were in the age group 20-29 years and in the study by Saung Oo et $\mathrm{al}^{4}$, majority of cases were in $26-30$ years age group which is comparable to our study (87\%). Also Saung Oo et al ${ }^{4}$ found that gestational age of $85.71 \%$ patients were in the range of 37 to 41 completed weeks, which is similar to our study ( $88 \%$ ). In the study by Roshdy et al ${ }^{5}$, mean gestational age of caesarean section was 38.6 weeks which is comparable to our study.

The most common indication was fetal distress in our study being $41 \%$, comparable to study by Roshdy et al ${ }^{5}$ who had fetal distress in $30 \%$ cases and Saung $\mathrm{Oo}^{4}$ et al who had fetal distress in $46.9 \%$ cases. Roshdy et al ${ }^{5}$ found failed induction of labour in $11.7 \%$ cases and Saung $\mathrm{Oo}^{4}$ et al in $6.2 \%$ cases comparable to our study $(9 \%)$. Chauhan et al ${ }^{6}$ reported that among patients with moderate to severe asthma, severe hypothyroidism, severe preeclampsia, post term or fetal growth-restricted fetuses with abnormal doppler studies, the risk of caesarean delivery for fetal distress was more than $20 \%$, a prevalence at least seven times more than the general population. Our hospital being a tertiary referral centre, the number of high risk patients is significantly higher and similar risk factors were observed in our study.

In the study titled "perinatal outcome in cesareans for fetal distress" by Gangwar et $\mathrm{al}^{7}$ maternal complications found associated with fetal distress were: antepartum hemorrhage, IUGR, oligohydramnios, hypertensive disorders of pregnancy, cord prolapse, meconium stained liquor and second stage arrest. In our study also similar risk factors were observed. In the study by Alharbi et al ${ }^{8}$ found preeclampsia in $11.8 \%$ cases comparable to our study (10\%). A study by David et al ${ }^{9}$ concluded that most emergency cesarean deliveries develop during labor in low-risk women and cannot be anticipated by prelabor factors and non-reassuring fetal heart rate is one of the major risk factors in these emergency cesareans. In our study, 39\% cases had no antepartum comorbidities and 25 $\%$ cases had no intrapartum risks factors. This also correlates with the study by Kohli et al ${ }^{3}$ who found that $27.78 \%$ of subjects undergoing caesarean for foetal distress had no risk factors. The percentage of primary LSCS done for fetal distress was $41 \%$.With the advent of continuous tocographic monitoring during labour, fetal distress due to meconium aspiration, cord traction, loop of cord around neck etc is being increasingly picked up and results in an early intervention to decrease both maternal and perinatal mortality and morbidity.

In an attempt to decrease the number of caesarean sections due to fetal distress American College of Obstetricians and Gynaecologist (ACOG) recommends replacement of the term fetal distress by non-reassuring fetal status followed by further description of findings fetal tachycardia or bradycardia, repetitive variable decelerations, late decelerations and low biophysical profile ${ }^{10}$. By specifying the cause for fetal distress, preventive and resuscitative measures can be undertaken to decrease caesarean section.

When additional coexisting risk factors are associated with the pregnancy as found in our study, the chances of complications are further increased. Hence it is essential to swiftly take action to avoid poor outcome, which is reflected as an increased rate of caesarean section.

\section{Conclusion}

The rates of caesarean section are increasing in all parts of the world. In this study, the most common indication for LSCS was fetal distress and oligohydramnios was the dominant antepartum cause. Preventive measures to reduce the risk factors such as regular blood pressure monitoring, early start of antihypertensive therapy, use of low dose aspirin for hypertensive disorders, hydration and rest in left lateral position for oligohydramnios and other such measures should be advocated.

\section{Conflict of interest: None. Disclaimer: Nil.}




\section{References}

1.WHO. WHO statement on caesarean section rates - 2015. Zeneva; 2017 [Accessed on December 3, 2017]. Available from: apps. who .int/iris/bitstream/ 10665/161442/ 1/WHO _RHR_15.02_eng.pdf

2.Ayres-de-Campos D, Spong CY, Chandraharan E. FIGO consensus guidelines on intrapartum fetal monitoring: Cardiotocography. Int J Gynecol Obstet. 2015; 131(1): 1324.

3.Kohli UA, Singh S, Dey M, Bal HK, Seth A. Antenatal risk factors in emergency caesarean sections done for fetal distress. Int J Reprod Contracept Obstet Gynecol. 2017; 6(6): 2421-6.

4.Saung Oo, Cheng HC, Yahya MS, Mooi CS. Risk factors for cesarean delivery in primigravida during spontaneous labor. Rawal Medical Journal. 2015; 40(4): 413-8.

5.Ahmed SR, Alsheeha MA, Alsammani MA. Risks and indications for cesarean sections in primiparous women: A case-control study. Ann Trop Med Public Health. 2013; 6: 541-4.

6.Chauhan SP, Magann EF, Scott JR, Scardo JA, Hendrix NW, Martin JN jr. Cesarean delivery for fetal distress: rate and risk factors. Obstet Gynecol Survey. 2003; 58(5): 33750 .
7.Gangwar R, Chaudhary S. Caesarean section for fetal distress and correlation with perinatal outcome. J Obstet Gynecol India. 2016; 66(S1):177-80.

8.Alharbi GA, Mosleh H, Alruhaili EM. Prevalence and causes associated with caesarean section among primi mothers in Madinah city, Saudi Arabia. World journal of pharmaceutical research. 2018; 7(15): 110-23.

9.David C, Lagrew MD, Melissa C, Bush MD, Anna M, McKeown MD, et al. Emergent (crash) caesarean delivery: Indications and outcomes. Am J Obstet Gynecol. 2006; 194(6): 1638-43.

10.ACOG. Inappropriate use of the terms Fetal distress and Birth asphyxia ACOG committee opinion no. 326. Americon College of Obstreticians and Gyneologists. Obstet Gynecol. 2005;106: 1469-70.

\footnotetext{
Preeti Malapure ${ }^{1}$, Sumayya ${ }^{2}$, Rajeshwari $\mathbf{P}^{3}$, Sreelatha $\mathbf{S}^{4}$

1 Junior Resident; ${ }^{2}$ Junior Resident; ${ }^{3}$ Junior Resident;

${ }^{4}$ Professor, Department of Obstetrics and Gynaecology, ESIC-MC-PGIMSR, Bangalore, Karnataka, India.
} 